\title{
Modelos microfinancieros latinoamericanos: una experiencia para la inclusión social y el desarrollo
}

\author{
Mario Luis Perossa*, Santiago Gigler ${ }^{\star *}$
}

\footnotetext{
Magíster en Finanzas. Profesor de grado y de posgrado, Universidad de Buenos Aires, y profesor titular e investigador, Universidad Maimónides, Buenos Aires, Argentina.

Correo electrónico:

marioperossa@yahoo.com.ar

* Licenciado en Economía, Becario de investigación programa PROPAI, Universidad de Buenos Aires, Buenos Aires, Argentina.

Correo electrónico:

giglersantiago@yahoo.com.ar
}

Recibido: 2 de febrero del 2015 Aprobado: 30 de abril del 2015

Cómo citar este artículo: Perossa, M. L. y Gigler, S. (2015). Modelos microfinancieros latinoamericanos: una experiencia para la inclusión social y el desarrollo. Cooperativismo \& Desarrollo, 23(106), 75-87. doi: http:// dx.doi.org/10.16925/co.v23i106.1124

\section{Resumen}

Introducción: en Latinoamérica, las instituciones microfinancieras han sido las encargadas de apalancar mediante pequeños préstamos a millones de individuos para financiar el capital de trabajo y la inversión de pequeñas máquinas. El objetivo del trabajo es mostrar los resultados que las microfinanzas arrojan en la mejoría de los indicadores de pobreza y en el crecimiento de la economía. Metodología: para el análisis cuantitativo, se utilizaron los datos de pobreza, crecimiento de los países medidos según su PBI, la tasa de ahorro, el crecimiento de empresas, entre otros indicadores de países de América Latina, los cuales fueron correlacionados entre sí para buscar las variables que podrían ser motoras o explicativas de la disminución de los índices de pobreza. Resultados: luego del análisis de correlación entre las distintas variables, se halló una relación fuerte entre el aumento de los montos promedio otorgados y la disminución de las tasas de pobreza; pudo probarse la profundización del mercado microcrediticio y su relación con la pobreza en los países analizados. Conclusiones: las microfinanzas se convirtieron en una herramienta poderosa de lucha contra la pobreza en los países que las practicaron, respetando la dignidad humana de aquellas personas que sufren de exclusión económica y social.

Palabras clave: ciclo virtuoso, círculo vicioso de la pobreza, instituciones microfinancieras, microcréditos, microfinanzas. 


\title{
Latin American Microfinancing Models: An Experience for Social Inclusion and Development
}

\begin{abstract}
Introduction: In Latin America, micro-finance institutions have been responsible for leveraging small loans to millions of individuals to finance working capital and investments in small machinery. The purpose of the work is to show the results that microfinance has on improving indicators for poverty and growth of the economy. Methodology: For the quantitative analysis, data were used on poverty, growth in countries according to their GNP, the savings rate and the growth of companies among other indicators in Latin American countries, which were then correlated with each other to examine variables that could be locomotives or explicative of the decrease in poverty rates. Results: After analyzing the correlation between the different variables, a strong relationship was found between the increase in the average amounts awarded and the decrease in the poverty rate; the strengthening of the microcredit market and its relationship to poverty in the countries that were analyzed was shown. Conclusions: Micro-finance became a powerful tool in the fight against poverty in the countries where it was practiced, respecting the human dignity of those individuals who suffer from economic and social exclusion.
\end{abstract}

Keywords: virtuous circle, vicious circle of poverty, micro-finance institutions, microcredit, micro-finance.

\section{Modelos microfinanceiros latino-americanos: uma experiência para a inclusão social e o desenvolvimento}

\section{Resumo}

Introdução: na América Latina, as instituições microfinanceiras têm sido encarregadas de levar, mediante pequenos empréstimos, milhões de indivíduos a financiarem o capital de trabalho e o investimento de pequenas máquinas. $\mathrm{O}$ objetivo deste trabalho é mostrar os resultados atingidos pelas microfinanças na melhoria dos indicadores de pobreza e no crescimento da economia. Metodologia: para a análise quantitativa, utilizaram-se dados de pobreza, crescimento dos países medidos segundo seu PIB, a taxa de poupança, o crescimento de empresas, entre outros indicadores de países latino-americanos, os quais foram correlacionados entre si para buscar as variáveis que poderiam ser motoras ou explicativas da diminuição dos índices de pobreza. Resultados: após a análise de correlação entre as diferentes variáveis, constatou-se uma relação forte entre o aumento das verbas médias outorgadas e a diminuição das taxas de pobreza; pôde ser provada a profundidade do mercado microcreditício e sua relação com a pobreza nos países analisados. Conclusões: as microfinanças se converteram numa ferramenta poderosa de luta contra a pobreza nos países que a praticaram respeitando a dignidade humana daquelas pessoas que sofrem de exclusão econômica e social.

Palavras-chave: ciclo virtuoso, círculo vicioso da pobreza, instituições microfinanceiras, microcréditos, microfinanças. 


\section{Introducción}

A principios de la década de 1970 , se llevaron a cabo programas experimentales en Bangladesh, Brasil y otros países, consistentes en pequeños créditos a grupos de mujeres pobres destinados a la financiación de inversiones para llevar adelante micronegocios. Este tipo de crédito otorgado a la formación de microempresas se basó en préstamos a grupos solidarios en los cuales cada miembro del grupo avala el pago del crédito de todos los miembros. Estos programas de préstamo a la microempresa se enfocaron casi exclusivamente en apoyar actividades generadoras de ingreso dirigidas a prestatarios muy pobres, siendo las mujeres el principal grupo de interesados.

En 1974, durante la preparación del Año Internacional de la Mujer, unas cien mujeres se reunieron en Ghana para debatir sobre los temas que las preocupaban, siendo el acceso al crédito la principal preocupación, por encima de la educación, la vivienda o la atención médica pues aseguraban que acceder al crédito les permitiría generar micronegocios, a través de los cuales podrían mejorar sus ingresos y de esta manera satisfacer sus necesidades. En la ciudad de México se realizó en 1975 la Conferencia de las Naciones Unidas sobre las Mujeres, donde un grupo de diez mujeres comenzó a planificar el Women's World Banking, que posteriormente abrió filiales en muchos países del mundo. De hecho, el Grameen Bank y muchas instituciones de microfinanzas de América Latina, África y Asia tienen entre las mujeres como a su principal grupo de clientes.

Según Mansell Carsten, citado por Padilla y Ulloa (2008), el acceso de los segmentos pobres de la población a los servicios financieros contribuye a su bienestar y al aprovechamiento de las oportunidades económicas disponibles en estos sectores. Este acceso importa, pero las microfinanzas no son el único camino para alcanzarlo. Bien conocida es la presencia habitual de agentes financieros informales (por ejemplo, prestamistas) o de mecanismos rotativos (por ejemplo, tandas) en estos estratos del mercado. En términos generales, se interpreta como microfinanzas la oferta de una o más prestaciones de toda una gama de servicios financieros proporcionados mediante la aplicación de tecnologías innovadoras de crédito y la prestación de otros servicios, que con las tecnologías bancarias tradicionales no podría hacerse rentable o sostenible; por lo tanto, las microfinanzas se definen como la prestación de servicios financieros a clientes de bajos ingresos incluyendo a los autoempleados.

Según el Banco Mundial (2000), la interpretación de la pobreza hasta finales de los noventa consistía en entenderla como la falta de activos de diferente índole (naturales, físicos, salud, financieros, etc.); por esta razón, las propuestas de política pública enfatizaban la necesidad de mejorar el acceso a educación, salud y empleo, y de políticas que aceleraran el crecimiento económico. Los resultados de la aplicación de estos lineamientos de política durante la década de los noventa demostraron que esto no era suficiente. Aunque el crédito por sí solo no es suficiente para impulsar el desarrollo económico, permite que los pobres adquieran su activo inicial y utilicen su capital humano y productivo de manera más rentable. Los pobres pueden además emplear los servicios de ahorro y seguro para planificar futuras necesidades de fondos y reducir el riesgo ante posibles variaciones en sus ingresos y gastos. En grados diferentes y en cada país hay personas que padecen de exclusión social y financiera.

El campo de las microfinanzas es de tan amplio espectro como las finanzas mismas. En él se insertan cuatro conceptos básicos que son los seguros o más conocidos últimamente como microseguros, el microcrédito, ahorro y las transferencias (Mena, 2005). Siguiendo a Padilla y Ulloa (2008), se trata de mecanismos innovadores de crédito y facilidades de ahorro destinados a aquella porción de la población excluida del sector financiero formal. Entonces es posible pensar el microcrédito como un instrumento, no el único, de lucha contra la pobreza, susceptible de ser utilizado desde distintas perspectivas que conducirán, lógicamente, a distintos resultados según sea la perspectiva que se elija.

En opinión de Lacalle, Rico, Márquez, Javo, Durán, Jiménez et al. (2010), existen cinco grupos de microcréditos que abarcan todos los ámbitos de actuación en los que los microcréditos se han desarrollado con mejor o peor fortuna. Cada uno de ellos implica una metodología particular, esto es, una estrategia operativa y de gestión propia para cada grupo (Lacalle et al., 2010). Los cinco grupos son:

1. Microcréditos contra la pobreza extrema (personas que viven con menos de un dólar al día).

2. Microcréditos para el desarrollo (personas pobres pero con sus necesidades básicas cubiertas).

3. Microcréditos para la inclusión (personas excluidas y marginadas; no necesariamente pobres). 
4. Microcréditos para emprender (personas con una idea de microactividad económica y que actualmente ya están recibiendo ingresos; o por trabajo o por subsidio.

5. Microcréditos para el empleo (personas muy emprendedoras que quieren poner en marcha un negocio formal con aspiración de crecer y generar empleo; figura 1).

La población antes mencionada, excluida de los servicios bancarios ofrecidos por las entidades comerciales habituales, cuenta con las características particulares que presenta el sector de la población de bajos ingresos: no pueden ofrecer garantías aceptables, presentan un alto riesgo crediticio y el monto de los créditos requeridos es demasiado pequeño para las instituciones que aspiran a ser rentables; por lo tanto, es aquí donde se pone el énfasis en aquellas innovaciones tecnológicas que permiten recabar y usar información, introducir incentivos de cumplimiento en los contratos de préstamo y hallar las maneras de hacerlos más apropiados a las características de los usuarios meta, de forma que esto permita el diseño de servicios financieros que respondan adecuadamente a las legítimas necesidades de estos demandantes, generalmente alejados de la economía formal y los mercados de trabajo registrados.

\section{Marco conceptual}

$\mathrm{Al}$ respecto, es necesario precisar la relación entre las microfinanzas y el crecimiento o desarrollo económico. Este se puede remontar a los conceptos de Joseph Schumpeter, en su libro Teoría del desenvolvimiento económico (1978), en el que plantea, entre otros, dos actores fundamentales para el desarrollo: el primero es el crédito y el segundo es el empresario. El microempresario es quien genera el progreso a través de una transferencia temporal de poder adquisitivo (microcrédito). Continuando con la idea de Schumpeter, correspondería relacionar las microfinanzas con el tercer caso de desarrollo económico, el de la apertura de un mercado, referido en el caso del microcrédito a la profundización del mercado a través de la inclusión de nuevos agentes que inducen a un aumento del tamaño del mercado. Por lo tanto, el crédito y el microempresario están positivamente relacionados con el mejoramiento de la economía y el ingreso de nuevos agentes al ciclo productivo. De esta manera, el desarrollo económico se encuentra influenciado por este nuevo y veloz sector de la economía, constituyendo una sólida alternativa para lograr la superación de la pobreza y la exclusión social.

Los microcréditos surgen para sacar del círculo vicioso de la pobreza a millones de personas muy pobres. En línea con el pensamiento de Schumpeter, es posible distinguir la teoría del círculo vicioso de la pobreza, enunciada por el economista Ragnar Nurkse (1955), que afirma que las personas más pobres están sumidas en un camino sin salida - un círculo vicioso-, del cual no pueden escapar. En la figura 2, es posible observar esta teoría. Según Nurkse, los pobres destinan sus pequeños ingresos enteramente al consumo, por lo que no tienen ninguna capacidad de ahorro ni de inversión, lo que a su vez limita las posibilidades de incrementar su renta futura. La única forma de romper este esquema de pobreza y convertirlo en un círculo virtuoso es mediante la inyección externa de capital (Lacalle et al., 2010).

El economista Ragnar Nurkse enunció, en su teoría sobre el círculo vicioso de la pobreza, que esta no puede erradicarse sin inyección de fondos adicionales, ya que los pobres no tienen capacidad de ahorro y, por lo tanto, no pueden incrementar su renta futura, pues todos los ingresos deben volcarlos al consumo para manutención. La transformación del círculo vicioso en un círculo virtuoso, según Nurkse, es a través de la inyección externa de capital de trabajo o inversión de pequeñas máquinas, que posibilitaría el apalancamiento por préstamos externos reembolsables.

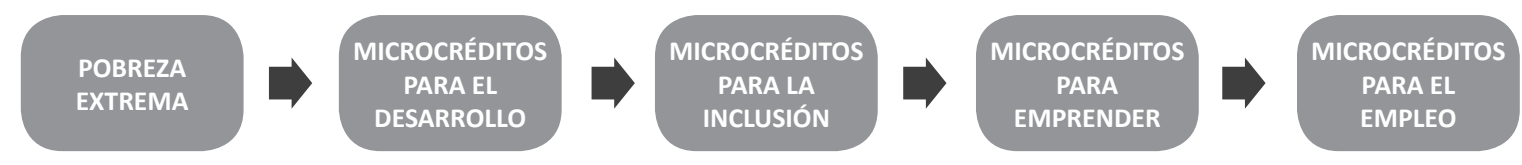

Figura 1. Escala de microcréditos. Elaboración propia basada en el Glosario básico sobre microfinanzas, por Lacalle et al., 2010. Los autores no consideran los microcréditos para la pobreza extrema, ya que se corresponde con carencias elementales que no significan inversión sino consumo. Generalmente, estos créditos o subsidios son no reembolsables y son otorgados por el Estado u organizaciones caritativas. 


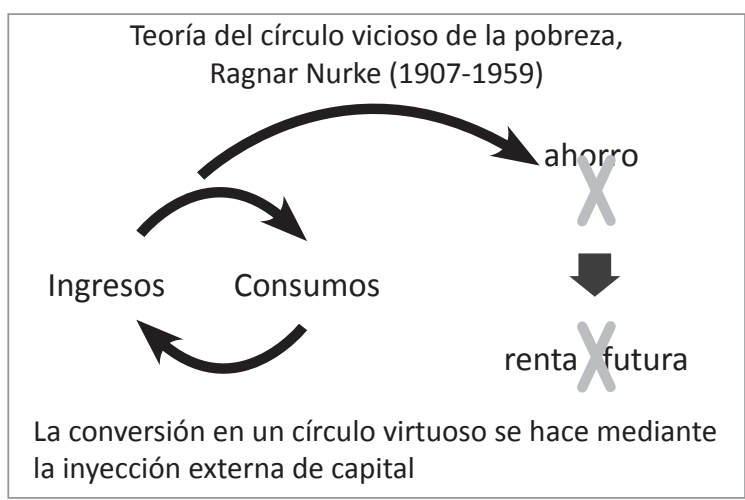

Figura 2. El círculo vicioso de la pobreza. Elaboración propia.

Muchas regiones del planeta aún continúan en la trampa del círculo vicioso de la pobreza y el subdesarrollo, afirmado en las condiciones sociales, económicas y políticas que impiden su expansión cultural y económica. Al analizar el funcionamiento del sistema es posible observar que en realidad existen tres círculos viciosos - cultural, demográfico y económico-financiero- que están interconectados y se realimentan mutuamente.

1. El círculo vicioso cultural: la falta de formación personal y la inadecuación de la cultura social impiden el desarrollo y el subdesarrollo impide la diseminación de la cultura.

2. El círculo vicioso demográfico: la alta natalidad y sobrepoblación produce miseria y la miseria impide el control de la natalidad.

3. El círculo vicioso económico-financiero: los bajos salarios determinan baja productividad laboral que impide el crecimiento de las rentas del trabajo.

De esta manera, pensando el problema de la exclusión desde una perspectiva distinta a la que la reduce a la pérdida de ingresos, es posible enriquecer el análisis del problema y, por consiguiente, las acciones necesarias para enfrentarlo. Así, se puede observar cómo los efectos de la exclusión -originada en los problemas en el mercado de trabajo- pueden llevar a un proceso de desafiliación en tanto ruptura de vínculos relacionales. $\mathrm{O}$, en un sentido aún más amplio, se puede entender la exclusión como una desigualdad en muchas dimensiones (aunque la exclusión del mercado de trabajo suele tener un efecto múltiple sobre las otras esferas): económica, social, política y cultural, entre otras. De este modo, una óptima utilización de los microcréditos será aquella que permita enfrentar la exclusión sin reducirla en su complejidad (Arnaiz, 2010).

\section{Las instituciones micro financieras (IMF)}

Para Larraín (2009), un elemento que ha servido para diferenciar los distintos modelos de microfinanzas (instituciones microfinancieras) en América Latina han sido los conceptos de upgrading y downscaling. El upgrading se refiere a la transformación de organizaciones de microfinanzas no gubernamentales (ONG) en entidades formales supervisadas por las autoridades bancarias (Berger, Otero y Schor, 2006). A su vez, el downscaling es el proceso a través del cual las instituciones financieras formales, tradicionalmente fuera del ámbito de las microfinanzas, se involucran en este sector (Marulanda, 2006).

Al nivel de país, Larrain (2009) considera que es posible contribuir al éxito de las IMF en la medida en que este reúna cuatro condiciones:

1. Que la cobertura y profundidad del mercado lo sitúe entre los países más desarrollados en este campo.

2. Que el entorno de negocios sea propicio para el desarrollo de este mercado.

3. Que las condiciones de acceso al crédito, medidas a través de las tasas de interés, sean competitivas.

4. Que exista una masa crítica de instituciones operando en el negocio de las microfinanzas, ya sean bancos, cooperativas, instituciones especializadas, etc.

El upgrading fue en las últimas dos décadas un factor fundamental para el desarrollo de las IMF en América Latina. Sibien las motivaciones para el upgrading son variadas, Larraín (2009) opina que quizás la más importante ha sido la búsqueda de ampliación de las fuentes de financiamiento para lograr a su vez un mayor volumen de préstamos. Ejemplos de esta categoría son Calpía en El Salvador, Banco ProCredit Los Andes en Bolivia, Chispa en Nicaragua, el Banco Sol de Bolivia, Finamérica de Colombia, Compartamos en México y MiBanco en Perú.

El interés por el downgrading se manifiesta en la rentabilidad del nicho de negocio y la diversificación de productos de las entidades ante una creciente competencia del mercado en las áreas tradicionales de las finanzas. Los representantes más significativos del downscaling son el Banco Agrícola de El Salvador, el Banco Solidario y Credife de Ecuador, el Banco Santander/Banefe de Chile, el Banco de Crédito de Perú, el Banco Caja Social de Colombia y también entidades estatales que entraron al sector, siendo las 
más emblemáticas el Banco do Nordeste de Brasil y el Banco Estado de Chile.

Los bancos comerciales de Latinoamérica también están dirigiendo su mirada hacia las microfinanzas. En algunos casos puntuales, las microfinanzas son su principal actividad, como en el caso de MiBanco en Perú o Banco ProCredit en Ecuador, mientras que para otros, se trata de un producto nuevo como en el Banco Bancafé en Honduras, el Banco Pichincha en Ecuador o el Banco Santander en Chile. En cierto modo, los bancos comerciales hacen un downscaling para poder servir al típico cliente microfinanciero. En el otro extremo del espectro de la regulación, se encuentran las instituciones financieras no reguladas, las cuales parecen enfocarse cada vez más en las zonas menos urbanizadas (Navajas y Tejerina, 2007).

Las ONG continúan siendo las principales IMF no reguladas, aunque también existen compañías privadas (con una clara participación de accionistas) que están surgiendo en mercados como el de Argentina. También existe una tercera tendencia de negocios denominada down-market, este último modelo cuenta con varias instituciones financieras de créditos de consumo que han sido pioneras en el otorgamiento de créditos a sectores de bajos recursos, lo que significa la entrada de entidades dedicadas a créditos para el consumo que se orientan a los segmentos de ingresos más bajos de la población. Un ejemplo del modelo down-market es el Banco Económico de Bolivia, el cual se dedicaba al consumo y luego incursionó en el sector microcrédito, aunque luego se retiró del segmento debido a una ola de sobreendeudamiento que afectó al sector. El down-market ha tenido éxito en el financiamiento de créditos de consumo a las personas pero ha presentado problemas en algunas entidades cuando se aplica el crédito a la microempresa, debido a las fuertes diferencias entre los modelos.

Resulta importante para la continuidad de la actividad el concepto de sostenibilidad, en el ámbito de las microfinanzas. Este concepto implica una serie de condicionantes y requisitos determinados, al menos desde el punto de vista teórico, que deben asumir e implementar las IMF. A este respecto, existe un cierto grado de consenso cuando se habla de sostenibilidades en relación a la actividad y a las instituciones microfinancieras, por lo cual es posible señalar algunas características (Cortés, 2008):

1. Subsidiación operativa de la actividad microfinanciera ordinaria;

2. Profesionalización de los agentes y de las instituciones microfinancieras;
3. Una dimensión adecuada que permita escalar la producción y obtener resultados positivos, permitiendo a su vez la continua capitalización de las entidades microfinancieras;

4. Una activa gestión del riesgo para este tipo de productos financieros o parafinancieros;

5. Cierto grado de institucionalización;

6. Regulación y existencia de un marco jurídico mínimo que permita garantizar el cumplimiento de los contratos;

7. Equilibrio entre ahorro e inversión crediticia en el ámbito local;

8. Asesoramiento financiero a los tomadores del crédito;

9. Una importante cantidad de instrumentos microfinancieros que permita cubrir la mayor parte de las necesidades financieras de las comunidades locales.

En los últimos años, los bajos niveles de riesgo y morosidad que tradicionalmente han mostrado las instituciones de microfinanzas se ven afectados por el sobreendeudamiento, la menor actividad económica y en cierta medida por la crisis financiera internacional originada en la crisis subprime y que aún no ha terminado. En algunos casos, se han registrado niveles inusuales en la morosidad, aunque transitorios, en otros el impacto ha sido leve, y en otros los niveles de mora se han más que duplicado (Fondo Multilateral de Inversiones, 2009). Como corolario de esta situación, es que muchas instituciones han decidido tomar determinadas acciones a efectos de reforzar su análisis de riesgo. Entre las más frecuentes figuran las siguientes:

1. Reforzamiento de procesos de evaluación, seguimiento y recuperación de cartera. Se ha sacrificado tiempo de desembolso por un mayor tiempo en el análisis, lo cual ha reducido la presión por colocar.

2. Reducción de montos y plazos, además del aumento en la frecuencia de pagos.

3. Refinanciación y reprogramación de deudas. Antes de castigar o enviar clientes a cobranza externa, algunas instituciones están buscando rescatarlos con medidas transitorias.

4. Ampliación de la jornada laboral para gestiones de cobro.

5. Instituciones con un horario de trabajo ya establecido lo han ampliado para dedicarse exclusivamente a cobranzas. 


\section{Metodología}

\section{Muestra}

Para la realización de la presente investigación, se recurrió a información pertinente a ciertos países de Latinoamérica. Particularmente, se tomaron datos de Bolivia, Brasil, Chile, Colombia, Guatemala, México, Perú. Estos 7 países analizados representan el 24\% sobre el total de países de América Latina y el Caribe (29 países).

La elección de estos países no ha sido casual, sino que corresponde a un análisis de datos sobre la presencia de instituciones volcadas al microcrédito, junto con el acceso y la existencia de información al respecto.

Principalmente, la información recogida corresponde a la evolución, tanto cualitativa como cuantitativamente, de los microcréditos en Latinoamérica por un lado, y cuestiones estructurales de la economía y la sociedad por el otro. Siendo la relación e impacto una cuestión importante del análisis.

La información relevante de las instituciones microfinancieras fue tomada de los informes presentados en los años 2009-2013 de Microfinanzas Américas: las 100 mejores, presentados por el Fondo Multilateral de Inversiones (Fonim), miembro del Banco Interamericano de Desarrollo (BID), y el Microfinance Information Exchange, Inc. (MIX). Para ello, se tomará la incidencia de los préstamos en relación con la variación de la tasa de pobreza.

Como puede observarse en la tabla 1, los créditos internos al sector privado crecieron de manera generalizada en la región, notando un periodo favorable para las inversiones y el clima de negocios. De la misma manera, y en línea con este periodo favorable, el registro de nuevas empresas, observado en la tabla 2 , fue positivo y ascendente en el periodo, reforzando la idea de fortaleza del sector privado.

Tabla 1

Crédito interno al sector privado (\% del PIB)

\begin{tabular}{|c|c|c|c|c|c|}
\hline \multirow{2}{*}{ País } & 2008 & 2009 & 2010 & 2011 & 2012 \\
\hline & $\%$ & $\%$ & $\%$ & $\%$ & $\%$ \\
\hline Bolivia & 34,69262457 & 37,0182158 & 40,342284 & 40,81778196 & 44,21753457 \\
\hline Brasil & 53,09576376 & 48,8671179 & 54,38481799 & 61,34992585 & 68,53573537 \\
\hline Chile & 100,1401374 & 102,3722397 & 99,25708201 & 101,5474023 & 104,3533516 \\
\hline Colombia & 37,72136389 & 39,96437771 & 43,70300555 & 44,71854688 & 48,83490012 \\
\hline Guatemala & 27,31264063 & 25,34157923 & 23,83047287 & 23,57236902 & 31,6648137 \\
\hline México & 20,83671543 & 22,77074217 & 24,31518855 & 25,71075389 & 27,54590984 \\
\hline Perú & 25,74956071 & 25,36129468 & 25,19368831 & 27,32853686 & 28,22564967 \\
\hline
\end{tabular}

Nota. Elaboración propia con base en datos del Banco Mundial.

Tabla 2

Nuevas empresas registradas (número)

\begin{tabular}{|c|c|c|c|c|c|}
\hline \multirow{2}{*}{ País } & 2008 & 2009 & 2010 & 2011 & 2012 \\
\hline & Empresas registradas & Empresas registradas & Empresas registradas & Empresas registradas & Empresas registradas \\
\hline Bolivia & 2448 & 2623 & 2737 & 3065 & 3541 \\
\hline Brasil & 56704 & 51717 & 66512 & 64476 & 53876 \\
\hline Chile & 36698 & 38870 & 44353 & 58407 & 68439 \\
\hline Colombia & 37810 & 38265 & 45273 & 56434 & 62993 \\
\hline Guatemala & 5476 & 5126 & 5222 & 5088 & 4306 \\
\hline México & 50392 & 60358 & 67648 & 65016 & 68666 \\
\hline Perú & 37079 & 38131 & 40694 & 70380 & 74145 \\
\hline
\end{tabular}

Nota. Elaboración propia con base en datos del Banco Mundial. El índice de empresas registradas se refiere a la cantidad de nuevas corporaciones de responsabilidad limitada registradas en el año calendario. 
Sin embargo, las transferencias recibidas por organismos internacionales, mostradas en la tabla 3, no acompañan esta tendencia. Es posible que el desarrollo de la región produzca un trade off respecto a la voluntad y al incentivo de ayuda por parte de estos organismos.

El crédito interno al sector privado se refiere a los recursos financieros otorgados al sector privado, por ejemplo, mediante préstamos, compra de valores que no constituyen una participación de capital y créditos comerciales y otras cuentas por cobrar, que crean un derecho de reembolso. En el caso de algunos países, estos derechos incluyen el crédito a empresas públicas.

La asistencia oficial neta para el desarrollo (AOD) comprende los desembolsos de préstamos en condiciones concesionarias (netos de reembolsos del principal) y las donaciones otorgadas por organismos oficiales de los miembros del Comité de Asistencia para el Desarrollo (CAD), por instituciones multilaterales y por países que no integran el CAD a fin de promover el desarrollo y el bienestar económico en los países y territorios que figuran en la lista del CAD de destinatarios de AOD. Incluye los préstamos que tienen un elemento de donación de al menos el 25\% (calculado a una tasa de descuento del 10\%).

Con respecto a la repercusión del crecimiento en el nivel de vida, tomaremos los datos de la tabla 4 y la tabla 5 para mostrar que a nivel general tuvo incidencia en la pobreza. Se podrá ver que la pobreza varía cuando varía el crecimiento del país y la distribución de los créditos otorgados. Si bien la incidencia no es nivelada entre los países, presenta un sesgo positivo en ellos a nivel general.

Tabla 3

Ayuda Oficial al Desarrollo (ODA) neta recibida (\% del INB)

\begin{tabular}{cccccc}
\hline \multirow{2}{*}{ País } & 2008 & 2009 & 2010 & 2011 & 2012 \\
\cline { 2 - 6 } & Ayuda Oficial & Ayuda Oficial & Ayuda Oficial & Ayuda Oficial & Ayuda Oficial \\
\hline Bolivia & 3,890643518 & 4,351626004 & 3,944419272 & 3,167660271 & 2,592436237 \\
Brasil & 0,028516404 & 0,021215391 & 0,021541077 & 0,033559408 & 0,058197115 \\
Chile & 0,064608131 & 0,04884234 & 0,097360937 & 0,069106826 & 0,049266446 \\
Colombia & 0,414497874 & 0,469915839 & 0,326725992 & 0,3180907 & 0,215171168 \\
Guatemala & 1,403263627 & 1,025277523 & 0,98069342 & 0,843201144 & 0,609950865 \\
México & 0,013802631 & 0,020864325 & 0,045201134 & 0,084266372 & 0,035808228 \\
Perú & 0,410371605 & 0,391055262 & $-0,186371089$ & 0,384728582 & 0,218510643 \\
\hline
\end{tabular}

Nota. Elaboración propia con base en datos del Banco Mundial.

Tabla 4

Crecimiento y cantidad de pobreza

\begin{tabular}{|c|c|c|c|c|c|c|c|c|c|c|c|c|}
\hline & Pobreza & Incidencias & Resultado & Pobreza & Incidencias & Resultado & Pobreza & Incidencias & Resultado & Pobreza & Incidencias & Resultado \\
\hline Chile & $-1,59 \%$ & $6,40 \%$ & $-0,10 \%$ & $-1,61 \%$ & $4,94 \%$ & $0,08 \%$ & $-1,64 \%$ & $4,48 \%$ & $-7,00 \%$ & $-1,67 \%$ & $4,42 \%$ & $-0,07 \%$ \\
\hline Colombia & $-3,74 \%$ & $31,68 \%$ & $-1,19 \%$ & $-7,50 \%$ & $28,62 \%$ & $-2,15 \%$ & $-9,01 \%$ & $28,72 \%$ & $-2,59 \%$ & $-6,27 \%$ & $23,72 \%$ & $-1,49$ \\
\hline Ecuador & $10,62 \%$ & $6,68 \%$ & $0,73 \%$ & $-10,00 \%$ & $7,49 \%$ & $-0,75 \%$ & $-22,67 \%$ & $3,85 \%$ & $-0,87$ & $-7,47 \%$ & $5,59 \%$ & $-0,42$ \\
\hline Guatemala & $0,00 \%$ & $0,00 \%$ & $0,00 \%$ & $0,00 \%$ & $0,00 \%$ & $0,00 \%$ & & & & $-12,86 \%$ & & $0,00 \%$ \\
\hline México & $6,62 \%$ & $37,48 \%$ & $2,48 \%$ & $6,21 \%$ & $40,34 \%$ & $0,03 \%$ & $0,22 \%$ & $44,30 \%$ & $0,10 \%$ & $0,22 \%$ & $48,26 \%$ & $0,11 \%$ \\
\hline Perú & $-16,14 \%$ & $17,56 \%$ & $-2,83 \%$ & $-6,10 \%$ & $18,62 \%$ & $-0,01 \%$ & $-10,00 \%$ & $18,65 \%$ & $-1,87 \%$ & $-7,78 \%$ & $18,00 \%$ & $-1,40 \%$ \\
\hline SUMAS & $0,00 \%$ & $100,00 \%$ & $-0,91 \%$ & $0,00 \%$ & $100,00 \%$ & $-0,02 \%$ & & $100,00 \%$ & $-5,30 \%$ & & $100,00 \%$ & $-3,27 \%$ \\
\hline
\end{tabular}

Nota. Tomado de "Relación entre el auge de los microcréditos financieros y el alivio de la pobreza en el escenario latinoamericano", por M. Perossa y A. Marianaro, (2014), en Revista Global de Negocios, 2(4), p. 25. Se muestran los resultados de la tasa de incidencia de la pobreza sobre la base de la línea de pobreza urbana, y el resultado del impacto del crecimiento de la cantidad de microcréditos otorgados. Fue elaborada a partir de los datos extraídos del Fondo Multilateral de Inversiones (2009). 
Tabla 5

Crecimiento y monto total de préstamos

\begin{tabular}{|c|c|c|c|c|c|c|c|c|c|c|c|c|}
\hline \multirow{2}{*}{ PAÍS } & \multicolumn{3}{|c|}{2008} & \multicolumn{3}{|c|}{2009} & \multicolumn{3}{|c|}{2010} & \multicolumn{3}{|c|}{2011} \\
\hline & Pobreza & Incidencia & Resultado & Pobreza & Incidencia & Resultado & Pobreza & Incidencia & Resultado & Pobreza & Incidencia & Resultado \\
\hline Chile & $-1,59 \%$ & $22,53 \%$ & $-0,36 \%$ & $-1,61 \%$ & $17,70 \%$ & $-0,29 \%$ & $-1,64 \%$ & $21,72 \%$ & $-0,36 \%$ & $-1,67 \%$ & $19,36 \%$ & $-0,32 \%$ \\
\hline Colombia & $-3,74 \%$ & $28,08 \%$ & $-1,05 \%$ & $-7,50 \%$ & $27,47 \%$ & $-2,06 \%$ & $-9,01 \%$ & $26,77 \%$ & $-2,41 \%$ & $-6,27 \%$ & $17,47 \%$ & $-1,10$ \\
\hline Ecuador & $10,62 \%$ & $11,93 \%$ & $1,27 \%$ & $-10,00 \%$ & $13,18 \%$ & $-1,32 \%$ & $-22,67 \%$ & $4,60 \%$ & $-1,04$ & $-7,47 \%$ & $9,75 \%$ & $-0,73$ \\
\hline Guatemala & $0,00 \%$ & $0,00 \%$ & $0.00 \%$ & $0,00 \%$ & $0,00 \%$ & $0,00 \%$ & $0,00 \%$ & $0,00 \%$ & $0,00 \%$ & $-12,86 \%$ & $0,00 \%$ & $0,00 \%$ \\
\hline México & $6,62 \%$ & $15,23 \%$ & $1,01 \%$ & $6,21 \%$ & $13,09 \%$ & $0,81 \%$ & $0,22 \%$ & $13,50 \% \%$ & $0,03 \% \%$ & $0,22 \%$ & $13,56 \%$ & $0,03 \%$ \\
\hline Perú & $-16,14 \%$ & $22,22 \%$ & $-3,59 \%$ & $-6,10 \%$ & $28,56 \%$ & $-1,74 \%$ & $-10,00 \%$ & $33,41 \%$ & $-3,34 \%$ & $-7,78 \%$ & $39,87 \%$ & $-3,10 \%$ \\
\hline SUMAS & & $100,00 \%$ & $-2,72 \%$ & & $100,00 \%$ & $-4,59 \%$ & & $100,00 \%$ & $-7,12 \%$ & & $100,00 \%$ & $-5,22 \%$ \\
\hline
\end{tabular}

Nota. Tomado de "Relación entre el auge de los microcréditos financieros y el alivio de la pobreza en el escenario latinoamericano", por M. Perossa y A. Marianaro, 2014, en Revista Global Finanzas, 2(4), p. 22. Se muestran los resultados de la tasa de incidencia de la pobreza sobre la base de la línea de pobreza urbana, y el resultado del impacto del crecimiento de la cantidad de microcréditos otorgados. Fue elaborada a partir de los datos extraídos del Fondo Multilateral de Inversiones (2009).

Tabla 6

Comercio (\% del PIB)

\begin{tabular}{cccccc}
\hline \multirow{2}{*}{ País } & 2008 & 2009 & 2010 & 2011 & 2012 \\
\cline { 2 - 6 } & \% del PIB & \% del PIB & \% del PIB & \% del PIB & \% del PIB \\
\hline Bolivia & 82,8670212 & 68,62707452 & 75,51162654 & 82,53030755 & 85,10394462 \\
Brasil & 27,13633384 & 22,11829707 & 22,77449978 & 24,5117143 & 26,62092387 \\
Chile & 81,04138041 & 66,76845041 & 69,81495546 & 72,8893252 & 68,42424529 \\
Colombia & 38,0520614 & 34,28000166 & 33,70084636 & 38,66886919 & 38,17723885 \\
Guatemala & 64,12502704 & 57,1057938 & 62,11508498 & 63,98391427 & 60,98251179 \\
México & 58,07108719 & 56,03479374 & 60,94653495 & 63,74599219 & 66,39214046 \\
Perú & 56,46560286 & 46,41854353 & 50,01229642 & 55,24166811 & 51,75004111 \\
\hline
\end{tabular}

Nota. Elaboración propia con base en datos del Banco Mundial, disponible en http://www.bancomundial.org/. El comercio es la suma de las exportaciones e importaciones de bienes y servicios, medidas como proporción del producto interno bruto.

De acuerdo con los datos analizados, es muy importante el PIB de los países a la hora del análisis. Tanto el monto como las cantidades de los créditos otorgados tendrán mucho que ver con el PIB ascendente de la región, por lo tanto influirá en la toma, la entrega y la disponibilidad de dinero para prestar, junto con un mercado en alza para explotar.

Al igual que el comercio, la formación bruta de capital plasmada en la tabla 7 presenta un comportamiento similar. No será constante y variará con los años, pero tendrá un comportamiento similar esa variación en los distintos países de la región.

\section{Resultados}

Para el análisis de correlación, se efectuaron dos mediciones diferentes: el índice que muestra la dis- minución de la pobreza para el grupo de países analizados, corregido por la incidencia de cada país de acuerdo con la cartera de créditos concedidos, por un lado, y el mismo índice corregido por el volumen total de préstamos concedidos. De esta forma, también es posible observar si el mayor beneficio se obtiene vía aumento de los montos otorgados y por el incremento de la cantidad de préstamos. Una decisión arbitraria que se tomó es la de medir el aumento de un año dado con la disminución de la pobreza medida el año siguiente, utilizando el supuesto de que dicha mejora no opera de forma inmediata, sino que los negocios necesitan un ciclo de al menos un año.

En la tabla 8, se pueden apreciar los resultados finales obtenidos para el caso del índice de pobreza afectado por la cantidad de contratos de préstamos y el aumento de la cantidad de préstamos, logrando una 
Tabla 7

Formación bruta de capital (\% del PIB)

\begin{tabular}{cccccc}
\hline \multirow{2}{*}{ País } & 2008 & 2009 & 2010 & 2011 & 2012 \\
\cline { 2 - 6 } & \% del PIB & \% del PIB & \% del PIB & \% del PIB & \% del PIB \\
\hline Bolivia & 17,55274034 & 16,97126133 & 17,00701824 & 19,5618916 & 17,6692172 \\
Brasil & 20,69445196 & 17,8380344 & 20,23861531 & 19,72626215 & 17,52253026 \\
Chile & 25,95906991 & 20,28333789 & 22,38398784 & 23,71001708 & 25,09450026 \\
Colombia & 23,48720128 & 22,44004225 & 22,12620476 & 23,87634015 & 23,92037761 \\
Guatemala & 16,4020252 & 13,05302191 & 13,93754897 & 15,22915701 & 14,95453774 \\
México & 24,40717547 & 22,87648033 & 22,02662166 & 22,2589076 & 23,1986876 \\
Perú & 27,46828639 & 20,8565553 & 25,174742 & 25,73309576 & 26,71440337 \\
\hline
\end{tabular}

Nota. Elaboración propia con base en los datos del Banco Mundial. La formación bruta de capital comprende los desembolsos en concepto de activos fijos (mejoramientos de terrenos, las adquisiciones de planta, maquinaria y equipo, y la construcción de carreteras, ferrocarriles y obras afines, y los edificios comerciales e industriales) e inventarios (existencias de bienes que las empresas mantienen para hacer frente a fluctuaciones temporales o inesperadas de la producción o las ventas)

Tabla 8

IMF de mejor desempeño: crecimiento del monto del préstamo en dólares

\begin{tabular}{cccccccc}
\hline & Bolivia & Brasil & Chile & Colombia & Guatemala & México & Perú \\
\hline Multiple R & 0,99114 & 0,78644 & 0,94386 & 0,96616 & 0,95738 & 0,65636 & 0,97863 \\
R Square & 0,98236 & 0,61848 & 0,89088 & 0,93347 & 0,91658 & 0,43081 & 0,95772 \\
Adjusted R Square & 0,97648 & 0,49131 & 0,8545 & 0,91129 & 0,88878 & 0,24108 & 0,94363 \\
Standard Error & 349657231,7 & 1626045610 & 869626476,9 & 679032235,5 & 769334703,3 & 1986105949 & 541279487,7 \\
Observations & 5 & 5 & 5 & 5 & 5 & 5 & 5 \\
\hline
\end{tabular}

Tabla 9

IMF de mejor desempeño: cantidad de préstamos

\begin{tabular}{|c|c|c|c|c|c|c|c|}
\hline & Bolivia & Brasil & Chile & Colombia & Guatemala & México & Perú \\
\hline Multiple R & 0,8039 & 0,12207 & 0,98663 & 0,9763 & 0,82651 & 0,83031 & 0,94889 \\
\hline R Square & 0,64626 & 0,0149 & 0,97344 & 0,95316 & 0,68312 & 0,68942 & 0,9004 \\
\hline Adjusted R Square & 0,52834 & $-0,31347$ & 0,96459 & 0,93755 & 0,57749 & 0,5859 & 0,8672 \\
\hline Standard Error & 1286854,3865 & 2147460,637 & 352586,3305 & 468264,2634 & 1217967,577 & 1205787,625 & 682,8421925 \\
\hline Observations & 5 & 5 & 5 & 5 & 5 & 5 & 5 \\
\hline
\end{tabular}

Tabla 10

Índices asociados de pobreza y microcréditos

\begin{tabular}{ccccccc}
\hline & $\mathbf{2 0 0 8}$ & $\mathbf{2 0 0 9}$ & $\mathbf{2 0 1 0}$ & $\mathbf{2 0 1 1}$ & Correlación & R2 \\
\hline Pobreza y cantidad de préstamo & $-0,91 \%$ & $-1,60 \%$ & $-5,30 \%$ & $-3,27 \%$ & & \\
Cantidad de prestamos & $36,36 \%$ & $14,20 \%$ & $17,87 \%$ & $22,96 \%$ & 0,4853 & 0,2355 \\
Pobreza y montos otorgados & $-2,72 \%$ & $-4,59 \%$ & $-7,12 \%$ & $-5,22 \%$ & 0,0558 \\
Monto de los prestamos & $30,16 \%$ & $17,18 \%$ & $26,03 \%$ & $23,55 \%$ & 0,2363 & 0 \\
\hline
\end{tabular}

Nota. Elaboración propia con base en datos obtenidos del Fondo Multilateral de Inversiones (2009). Los datos para los años analizados corresponden al 2008, 2009, 2010 y 2011 para los índices elaborados de "Cantidad de préstamos otorgados" y "Monto de los préstamos". Para los indicadores "Pobreza y cantidad de préstamos" y "Pobreza y montos otorgados", se tomaron aquellos que correspondes a un año posterior $(2009,2010,2011$ y 2012), ya que arbitrariamente los autores adjudican un tiempo de un año para los microcréditos permitan aliviar la situación de los prestatarios. 
correlación de 0,4853 , con un $\mathrm{R}^{2}=0,055$. Por otra parte, en la tabla 5 , se observa el mismo indicador de pobreza pero en este caso incidido por el volumen total de préstamos concedidos, para esta alternativa el coeficiente de correlación es de 0,2363 y un $\mathrm{R}^{2}=0,2355$.

El resultado positivo de la correlación entre las variables pobreza y profundización de las microfinanzas, en cualquiera de las dos dimensiones tratadas en la tabla 9, con la cantidad de préstamos, como en la tabla 10 con las microfinanzas, indica el carácter beneficioso para las clases humildes del uso intenso y correcto de esta herramienta. El análisis no solo permite corroborar la hipótesis planteada originalmente, sino también confirmar la teoría enunciada por Ragmar Nurkse, que explica que el círculo vicioso de la pobreza es posible transformarlo en un círculo virtuoso mediante la inyección de capital externo. Una mayor correlación entre el índice de la pobreza y la cantidad de créditos otorgados. Explica, además, que es más beneficioso llegar a más personas que aumentar significativamente el monto unitario de los préstamos.

\section{Discusión, conclusión e implicaciones}

En este artículo, las microfinanzas fueron definidas como un conjunto de instrumentos y de políticas destinadas a satisfacer las demandas financieras de ciertos grupos de interés que no poseen las características necesarias para el acceso al mercado comercial de crédito, para satisfacer sus necesidades de capital de trabajo y la adquisición de pequeñas máquinas. Los autores opinan que los microcréditos en particular y las microfinanzas en general son las herramientas adecuadas para brindar a los sectores marginados de la sociedad una posibilidad real de aumentar sus ingresos, lo cual, asociado con una mejor nutrición, una mayor educación y experiencia y la posibilidad de la realización personal, crean las condiciones necesarias para lograr la movilidad social y la plena integración de sus miembros a la sociedad.

En las tablas 10 y 11, se analiza cómo se correlacionan los montos y las cantidades de préstamos, con el desempeño de las mejores instituciones financieras. El resultado en ambas tablas es similar y lógico: los países no reaccionan de la misma forma al desempeño de las instituciones financieras. Esto es por rigideces y particularidades de las economías de cada nación y, por supuesto, por la presencia e influencia de estas en la economía en general.

Finalmente, la tabla 12 muestra el conjunto de resultados estadísticos obtenidos de los países analizados anteriormente.

Tabla 11

IMF de mejor desempeño: crecimiento del monto del préstamo en dólares

\begin{tabular}{ccccccccc}
\hline Regression Statistics & Bolivia & Brasil & Chile & Colombia & Guatemala & México & Perú \\
\hline Multiple R & 0,99114 & 0,78644 & 0,94386 & 0,96616 & 0,95738 & 0,65636 & 0,97863 \\
R Square & 0,98236 & 0,61848 & 0,89088 & 0,93347 & 0,91658 & 0,43081 & 0,95772 \\
Adjusted R Square & 0,97648 & 0,49131 & 0,85450 & 0,91129 & 0,88878 & 0,24108 & 0,94363 \\
Standard Error & 349657231,7 & 1626045610 & 869626476,9 & 679032235,5 & 760334703,3 & 1986105949 & 541279487,7 \\
Observations & 5 & 5 & 5 & 5 & 5 & 5 & 5 \\
\hline
\end{tabular}

Tabla 12

IMF de mejor desempeño: cantidad de préstamos

\begin{tabular}{cccccccc}
\hline Regression Statistics & Bolivia & Brasil & Chile & Colombia & Guatemala & México & Perú \\
\hline Multiple R & 0,80390 & 0,12207 & 0,98663 & 0,97630 & 0,82651 & 0,83031 & 0,94889 \\
R Square & 0,64626 & 0,01490 & 0,97344 & 0,95316 & 0,68312 & 0,68942 & 0,90040 \\
Adjusted R Square & 0,52834 & $-0,31347$ & 0,96459 & 0,93755 & 0,57749 & 0,58590 & 0,86720 \\
Standard Error & 1286854,386 & 2147460,637 & 352586,3305 & 468264,2634 & 1217967,577 & 1205787,625 & 682,8421925 \\
observations & 5 & 5 & 5 & 5 & 5 & 5 & 5 \\
\hline
\end{tabular}




\section{Análisis de los países implicados en el estudio}

1. Brasil: en el periodo analizado (2008-2012), se visualiza que las IMF de mayor desempeño presentaron un aumento tanto en dólares como en la cantidad de préstamos otorgados. En el país, se ve una tendencia a aumentar el PIB en los años analizados. Cuando se lo regresa con los montos de las instituciones microfinancieras principales, tiene una relación muy positiva, arroja un R2 del 0,9823, un número bastante alto y posiblemente explicativo de la situación de mejora en las instituciones por el PIB. Por otro lado, al analizar la tasa de pobreza junto con la variación en la cantidad de montos otorgados se observa que no se cuenta con los datos suficientes: si bien se puede establecer una relación entre los dos primeros años, luego deja de haber datos, siendo sesgada la regresión y explicando el no tan buen R2 de 0,64625.

Con respecto al aumento del monto de los créditos otorgados, al relacionarlos con la variación del PIB del país, se observa una tendencia al aumento de ambos importes; sin embargo, la regresión presenta un R2 de 0,618481341, de forma que no se puede establecer la explicación de una variable sobre la otra directamente. En cuanto a la cantidad de créditos y pobreza considerada "Number of poor at $\$ 5$ a day (PPP) (millions)" del Banco Mundial se observa una relación entre la variación de la pobreza y la cantidad de créditos que se otorgaron. Sucede algo similar al PIB. Si bien comparte una tendencia, mientras más aumentan los créditos (también está aumentando el PIB), más cae la pobreza. Una variable no es explicativa de la otra, ya que presenta un R2 de 0,014900916.

2. Chile: realizando los mismos análisis anteriores, muestra resultados más convincentes. Tanto los montos de los prestamos como el PIB creció, y parecen ser explicativos (R2 0,8908); como también la tasa de pobreza y la cantidad de préstamos (R2 0,9734); cabe destacar que se cuenta con todos los datos, por eso, posiblemente, los R2 den más altos y sea más consistente el resultado.

3. Colombia: se comporta de manera similar que en Chile, con todos los datos; existe una consistencia explicativa fuerte $(0,9334$ y 0,9531 respectivamente).

4. Guatemala: con respecto al PIB y los montos, presenta una explicación bastante robusta (R2 0,9165), sin embargo, los datos sobre la pobreza solamente se tienen a partir del 2011.
5. México: es un país destacable; en primer lugar, se cuenta con todos los datos que se desean analizar, por lo que no habría un sesgo en cuanto a ello. Lo llamativo es que si bien existe la tendencia de crecimiento del PIB, esta no es muy explicativa con respecto a los préstamos otorgados. Asimismo, la tasa de pobreza presenta un dato destacable, no baja continuamente como en todos los demás países, sino que hay años en los que se incrementa. De esta manera, no acompaña ni explica los montos otorgados.

6. Perú: sucede de manera muy similar a Colombia y Chile. Bajan las tasas de pobreza y aumenta el PIB. Los test presentan una explicación bastante sólida (R2 0,9577 y 0,9003 respectivamente).

El rol de los microcréditos en la disminución de la pobreza tiene un peso importante, pero podría deducirse que principalmente se ve afectado por el dinamismo de la economía en general. En época de crecimiento en muchos de los países analizados, es importante el sostenimiento de la red de microempresas para dar ese viento a favor y de esta manera posibilitar que la economía en general cuente con la capacidad de ampliar la producción e inversión no solo a los capitales ya existentes, sino expandiéndose y generando un mercado más competitivo.

La atención a los más pobres no debe ser contradictoria con el enfoque de comercialización que caracteriza el desarrollo de las entidades en Latinoamérica. Sin embargo, sí es claro que el microcrédito solo puede ser ofrecido a aquellos segmentos que tengan una mínima capacidad de pago y no debe ser utilizado como sustituto de los instrumentos de política social, los cuales deben continuar siendo responsabilidad de los gobiernos.

La evidencia disponible sugiere que las ONG logran focalizar mejor su atención en créditos de menor tamaño, seguidas por las microfinancieras y luego por los bancos comerciales. Sin embargo, el crédito promedio no es necesariamente un buen indicador de la atención efectiva de los segmentos de menores ingresos, lo cual hace necesario realizar mayores esfuerzos en la investigación relacionada con ese tema. De las cifras e investigaciones de la pobreza realizadas en Latinoamérica, se obtiene que la pobreza en términos de población se concentra en las zonas urbanas, pero es mayor en las áreas rurales, en términos cualitativos.

Por otra parte, en materia regulatoria y de supervisión es fundamental avanzar en el análisis y en 
la reforma de las normas que dificultan, encarecen o impiden el suministro de los demás servicios financieros (transferencias, depósitos, seguros) a los segmentos más pobres, informales y ubicados en lugares apartados, a efectos de no entorpecer el desarrollo del sector.

Una limitación para el presente estudio está dada por los escasos datos sobre los resultados de las políticas públicas de los países analizados y su incidencia en los indicadores, tanto de pobreza como de $\mathrm{PBI}$, aunque en este último caso es probable que este sea marginal. Esta misma limitación significa la posibilidad de abrir nuevas líneas de investigación para futuros trabajos sobre los resultados de las microfinanzas y su medición, separando el rol de los microcréditos como elemento dinamizador de la economía e impulsor de la movilidad social de los subsidios o préstamos para la subsistencia.

\section{Referencias}

Arnaiz, C. (2010). El sector de las Microfinanzas en Argentina: Balance, situación actual y perspectivas, Diplomatura en Desarrollo Local y Economía Local, FLACSO. Recuperado de http://observatorioess.org.ar/ blog/2011/08/

Berger, M., Otero, M. y Schor, G. (2006). Pioneers in the commercialization of microfinance: the significance and future of upgraded microfinance institution. En M. Berger, L. Goldmark y T. Miller-Sanabria. (Eds.), An Inside View of Latin American Microfinance. Washington: Inter-American Development Bank. Recuperado de http://idbdocs.iadb.org/wsdocs/getdocument. aspx?docnum $=1577359$

Cortés, F. (2008). Las microfinanzas: caracterización e instrumentos. Barcelona: Cajamar.

Fondo Multilateral de Inversiones. (2009). Microfinanzas Américas: las 100 mejores. Nueva York: Banco Interamericano de Desarrollo. Recuperado de http:// www.themix.org/sites/default/files/Microfinanzas\%20 Americas\%20100\%202009\%20ES.pdf
Lacalle, M., Rico, S., Márquez, J., Javo, B., Durán, J. Jiménez, I., De la Orden, M. et al. (2010). Glosario básico sobre microfinanzas. Madrid: Foro Nantik Lum de MicroFinanzas, Universidad Pontificia Comillas, Universidad Autónoma de Madrid.

Larraín, C. (2008). ¿Existe un modelo de microfinanzas en América latina? Cepal, Serie Financiamiento del desarrollo, 220. Recuperado de http://bivica.org/upload/ modelo-microfinanzas.pdf

Marulanda, B. (2006). Downscaling: moving Latin Amerian Banks into Microfinance. En M. Berger, L. Goldmark y T. Miller-Sanabria. (Eds.), An Inside View of Latin American Microfinance. Washington: Inter-American Development Bank. Recuperado de http:// idbdocs.iadb.org/wsdocs/getdocument.aspx?docnum $=1577359$

Mena, B. (2005). Microcréditos: un medio efectivo para el alivio de la pobreza. Buenos Aires: Universidad $\mathrm{Na}-$ cional de Quilmes. Recuperado de http://biblioteca. municipios.unq.edu.ar/modules/mislibros/archivos/ Microfinanzas\%20en\%20Argentina.pdf

Navajas, S. y Tejerina, L. (2007). Las microfinanzas en América Latina y el Caribe: ¿Cuál es la magnitud del mercado? Washington, D. C.: Banco Interamericano de Desarrollo. Recuperado de http://smye. info/gia-mexico/wp-content/uploads/2009/09/ Las-microfinanzas-en-America-Latina-y-el-Caribe-Cual-es-la-magnitud-del-mercado.pdf

Nurkse, R. (1955). Problemas de formación de capital en los países insuficientemente desarrollados. México: Fondo de Cultura Económica.

Padilla, F. y Ulloa, M. (2008). Investigación de gabinete sobre el estado de las microfinanzas a nivel local e internacional y su relación con el FOMMUR. México: PRONAFIN.

Perossa, M. L. y Marinaro, A. (2014). Relación entre el auge de los microcréditos financieros y el alivio de la pobreza en el escenario Latinoamericano. Revista Global de Negocios, 2(4), 15-24. Recuperado de M http://www. theibfr.com/ARCHIVE/RGN-V2N4-2014.pdf

Schumpeter, J. (1978). Teoría del desenvolvimiento económico. México: Fondo de Cultura Económica. 